\title{
Validity of photographs for food portion estimation in a rural West African setting
}

\author{
L Huybregts $^{1}$, D Roberfroid ${ }^{2}$, C Lachat ${ }^{1,2}$, J Van Camp ${ }^{1}$ and P Kolsteren ${ }^{1,2, *}$ \\ 'Department of Food Safety and Food Quality, Coupure Links 653, Ghent University, B-9000 Ghent, Belgium: \\ ${ }^{2}$ Child Health and Nutrition Unit, Department of Public Health, Prince Leopold Institute of Tropical Medicine, \\ Nationalestraat 155, B-2000 Antwerp, Belgium
}

Submitted 9 January 2007: Accepted 6 May 2007: First published online 9 August 2007

\begin{abstract}
Objective: To validate food photographs for food portion size estimation of frequently consumed dishes, to be used in a 24-hour recall food consumption study of pregnant women in a rural environment in Burkina Faso. This food intake study is part of an intervention evaluating the efficacy of prenatal micronutrient supplementation on birth outcomes.

Subjects: Women of childbearing age (15-45 years).

Design: A food photograph album containing four photographs of food portions per food item was compiled for eight selected food items. Subjects were presented two food items each in the morning and two in the afternoon. These foods were weighed to the exact weight of a food depicted in one of the photographs and were in the same receptacles. The next day another fieldworker presented the food photographs to the subjects to test their ability to choose the correct photograph.

Results: The correct photograph out of the four proposed was chosen in 55\% of 1028 estimations. For each food, proportions of underestimating and overestimating participants were balanced, except for rice and couscous. On a group level, mean differences between served and estimated portion sizes were between $-8.4 \%$ and $6.3 \%$. Subjects who attended school were almost twice as likely to choose the correct photograph. The portion size served (small vs. largest sizes) had a significant influence on the portion estimation ability.

Conclusions: The results from this study indicate that in a West African rural setting, food photographs can be a valuable tool for the quantification of food portion size on group level.
\end{abstract}

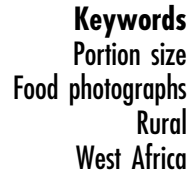

Keywords

hotographs

West Africa
The characterisation of a population's diet can be done by an array of dietary assessment methods. Dietary recalls, food-frequency questionnaires (FFQs), dietary records and food diaries all have in common that a large part of the random error is caused when estimating the portion size $^{1}$. Weighing served portions is therefore often taken as the gold standard. However, in developing countries like Burkina Faso, the lack of skilled workers, budgetary limitations, the low educational level of the subjects and the vastness of the country make the weighing method very hard to implement as a dietary assessment tool ${ }^{2}$.

As an alternative to weighing the food portion subjects consume, the portion size reported through FFQs or 24-hour dietary recalls are estimated by using portion size estimation aids. The selection of such an aid depends on several factors such as the setting of the study, population and budget restrictions. Cypel et al. ${ }^{1}$ reviewed the different portion size measurement aids and divided them into three-dimensional and two-dimensional aids. The first include household measures, real food samples, food replicates and food models. The latter comprise drawings of real foods/household measures/abstract shapes, food photographs, computer graphics and food package labels. Food photographs have the advantages of being easily adaptable to local conditions, cheap, reproducible and transportable. The use of photographs for food consumption assessment has been described in various studies $^{3-13}$, but almost entirely in industrialised and welleducated populations. Only Venter et al. ${ }^{13}$ evaluated the effectiveness of food photographs in urban health centres in South Africa.

The objective of the present study was to assess the ability of subjects to estimate consumed portion sizes with the aid of food photographs in a West African rural setting, which is characterised by a low educational level and the rarity of pictorial representations in the everyday environment. 


\section{Material and methods}

\section{Subjects and setting}

The study was undertaken in two villages, Karaba and Koho, which are part of the health district of Houndé in the province of Tuy, Burkina Faso. Houndé is one of the three rural districts in the health region of BoboDioulasso. It covers approximately $5000 \mathrm{~km}^{2}$ in the province of Tuy and is located on either side of the main road joining the two largest cities in Burkina Faso, Ouagadougou and Bobo-Dioulasso. In 2004, the estimated population was 231801 inhabitants. The two villages are the intervention area of a double-blind, randomised, placebocontrolled trial (MISAME) which investigates the effect of a multivitamin-minerals supplementation during pregnancy on birth outcomes.

A convenience sample of pregnant and non-pregnant women of childbearing age (15-45 years) was selected for this validation study. The minimum postulated sample size per evaluated food was fixed at 110 subjects by analogy with validation studies of FFQs ${ }^{14}$. This number was increased by $20 \%$ to 137 subjects to compensate for dropouts. For logistical reasons the study was carried out in two groups. Due to field labour, women were only at home in the morning and in the late afternoon. It was judged unrealistic to offer four foods per meal for consumption. Additionally it was difficult to retain the same group of women for a second session because during this period women often stayed for a longer period of time on the fields. A first group of 137 subjects evaluated the photographs of four foods; a second group of 137 persons evaluated the photographs of four other foods on a different day. The served portion sizes were randomly attributed to the subjects. Both groups were equally composed of women from both villages.

\section{Development of food photographs}

The diet of rural households in Burkina Faso is characterised by a high and frequent consumption of cereals as staple foods. The typical meal contains a cereal staple food accompanied by a sauce of leaves or groundnuts ${ }^{15}$.

An experimental food photograph album was compiled containing pictures of stiff cereal porridge, gruel, rice, couscous, rice with beans, liquid sauce, leafy sauce, and balls prepared from bean leafs. The foods were depicted on a plate except for the sauces and the gruel, which were depicted in a small bowl. The used plates and bowls were commonly available in both of the villages where the study was performed. The choice of the food items was based on the frequency of consumption during previously conducted 24-hour dietary recalls. Foods that come in discrete units such as bananas, biscuits, etc. were not included in the photograph album because they can be counted; drinks were not included because of their fixed price-quantity relationship. For each food four coloured printed photographs were included $(160 \mathrm{~mm} \times 100 \mathrm{~mm})$. The photographs were taken using a digital camera with 4.0 mega pixel resolution.

Standard portion sizes do not exist in Burkina Faso; therefore the choice of the portion sizes depicted was based on a previous small-scale food intake study on a sample of 34 women of childbearing age ( $15-45$ years) in the same region, who used a weighed record to estimate portion sizes (unpublished). It was first attempted to determine the depicted portion sizes as a function of the average intake from the small-scale food intake study, i.e. photos 2 and 3 at 0.5 standard deviations (SD) and photos 1 and 4 at 1.5 SD of the average intake of a food. However, the differences between the depicted portion sizes of some foods were widened if, during pre-testing, the differences between the photos were not distinguishable clearly enough for the subjects. The stiff cereal porridge, rice, couscous, rice with beans and balls from bean leafs were photographed on a plastic plate. Gruel, liquid sauce and leafy sauce were photographed in a plastic bowl. The portion served corresponded exactly to the portion size depicted on the food photographs.

The weight of the portion size in each photograph and examples of the food photographs used are given in Appendix A and B, respectively.

\section{Pre-testing the food photographs}

An experimental album of food photographs was pre-tested in both villages on an average sample of 23 subjects per food item. From this pre-test it was concluded that the way the stiff cereal porridge was presented on the photographs was creating confusion among the participants (mean group difference was $267 \pm 298 \mathrm{~g}$ for $n=64, P<0.0001)$. Initially, the stiff cereal porridge was presented in amorphous pieces which resulted in respondents counting the pieces depicted to compare with the number of pieces they ate the day before and not looking at the depicted quantity. For that reason the photographs for stiff cereal porridge were modified. The stiff cereal porridge was first solidified in a bowl and then depicted in quadrants (1, 2, 4 or 6$)$.

\section{Design}

In order to imitate free-living conditions, this study was performed at people's homes over eight locations spread over the two villages. The receptacles on which the foods were served were the same as depicted in the food photographs. The participants were invited in the morning and in the afternoon and were served two foods on both occasions. Four different portion sizes were weighed and served by fieldworkers for each food. Portion sizes were randomly allocated to each incoming participant, following a pre-established random list of portion sizes. Portions were weighed with a precision of 
$1 \mathrm{~g}$ with a Tefal ${ }^{\circledR}$ Origin 5 kitchen balance. The procedure and objectives of this study were explained to the participants and their oral consent was obtained. The next day, the participants were asked to estimate the portion size of the four foods served the previous day (morning and afternoon) with the aid of food photographs during individual house visits. To avoid interviewer bias, the interviewer with the food photographs was different from the one who served the foods.

Fieldworkers emphasised in their explanations that the participants had nine possible choices: choosing one of the four photographs; choosing between the photographs; choosing smaller than the smallest or larger than the largest depicted portion size. Additional data collected included school attendance and pregnancy status.

\section{Data analysis}

All statistical analysis was performed using Intercooled Stata version 8 software (StataCorp LP).

The percentages of portions correctly identified, overestimated and underestimated with the photographs were calculated for each subject and each food. A $\chi^{2}$ test for independence was used for proportions. A weighted $\kappa$ coefficient was used to analyse the pattern of agreement between the served and estimated portion sizes ${ }^{16}$. Quadratic weights for four categories were calculated: a disagreement by one photo was attributed a weight of 0.89 , by two photos a weight of 0.56 and a disagreement by more than two photos a weight of 0 . Because the data were ordinary, a Wilcoxon matched-paired signed rank test with a significance level of 0.05 was used to compare the mean estimated weight of portion sizes of each food against the actual weight of each portion. For the portion size in between two photographs, the average weight of the two neighbouring photographs was chosen. For estimations smaller or larger than the range of four photographs, half a class width was subtracted or added respectively.

To assess the importance of portion sizes, pregnancy status, village and school attendance on the risk of misclassification, logistic regression modelling was performed. The dependent variable was 'good classification of photos'.
The repeated measurements design (four foods per participant) was accounted for (CLUSTER command in Stata). All of the variables were included in the initial model. A backward procedure was applied to remove variables with $\alpha>0.05$ at the likelihood ratio. The quality of the model was evaluated with the Hosmer-Lemeshow $\chi^{2}$ test.

\section{Results}

\section{Sample characteristics}

As 17 subjects were lost to follow-up, data were available for 257 subjects. Fifty-one per cent lived in Karaba and 49\% in Koho. Of these 257 participants, 77 (30\%) were pregnant.

When evaluating educational level, 20.2\% of the women reported being able to read and write, whereas $79.0 \%$ reported to have never been to school. Only $5.4 \%$ of the women reported to have benefited from more than 6 years' schooling. Moreover it was noticed that school attendance was significantly higher in Karaba than in Koho (26.7\% vs. $15.1 \%, P<0.001)$.

\section{Evaluation of portion size estimation on individual level}

Table 1 shows the proportions of subjects who chose the photograph that corresponded with the previously served portion size. In $1.9 \%$ of the estimations subjects chose a portion size outside the range of the photos or between two photos. The results show that portion size was correctly estimated in $37.6 \%$ of cases for couscous to $92.0 \%$ for stiff cereal porridge. If adjacent photos are included these percentages increase from $85.5 \%$ for rice and beans to $97.6 \%$ for stiff cereal porridge. Kappa statistics were used to analyse the pattern of agreement between the served and estimated portion sizes. Modest agreement was achieved for rice, rice and beans, and liquid sauce, whereas substantial agreement was obtained for couscous, bean leaf balls and gruel. For stiff cereal porridge and leafy sauce an almost perfect agreement was obtained. All $\kappa$ statistics were statistically significant

Table 1 Percentage of participants estimating the portion size correctly, overestimating or underestimating, with kappa agreement statistics

\begin{tabular}{|c|c|c|c|c|c|c|c|}
\hline Food item & $n$ & $\begin{array}{l}\text { Correct } \\
\text { photo (\%) }\end{array}$ & $\begin{array}{l}\text { Underestimating with } \\
\text { one photo or less (\%) }\end{array}$ & $\begin{array}{l}\text { Overestimating } \\
\text { with one photo } \\
\text { or less }(\%)\end{array}$ & $\begin{array}{l}\text { Underestimating } \\
\text { with more than one } \\
\text { photo }(\%)\end{array}$ & $\begin{array}{l}\text { Overestimating } \\
\text { with more than } \\
\text { one photo }(\%)\end{array}$ & $\kappa$ \\
\hline Stiff cereal porridge & 125 & 92.0 & 3.2 & 2.4 & 0.8 & 1.6 & 0.92 \\
\hline Rice & 132 & 41.0 & 34.0 & 15.9 & 5.3 & 3.8 & 0.60 \\
\hline Rice and beans & 132 & 42.4 & 24.2 & 18.9 & 10.7 & 3.8 & 0.52 \\
\hline Couscous & 125 & 37.6 & 33.6 & 20.0 & 6.4 & 2.4 & 0.65 \\
\hline Bean leaf balls & 125 & 50.4 & 21.6 & 20.8 & 6.4 & 0.8 & 0.70 \\
\hline Sauce, leafy & 125 & 64.8 & 13.6 & 17.6 & 0.8 & 3.2 & 0.84 \\
\hline Sauce, liquid & 132 & 51.5 & 13.6 & 22.0 & 5.3 & 7.6 & 0.59 \\
\hline Gruel & 132 & 65.2 & 15.9 & 13.6 & 3.8 & 1.5 & 0.76 \\
\hline
\end{tabular}

Agreement levels ${ }^{16}$ : poor, $\kappa \leq 0$; slight, $0.01 \leq \kappa \leq 0.20$; modest, $0.21 \leq \kappa \leq 0.40$; moderate, $0.41 \leq \kappa \leq 0.60$; substantial, $0.61 \leq \kappa \leq 0.80$; almost perfect, $0.81 \leq \kappa \leq 1$. 
$(P<0.001)$. Overall, the correct photo was chosen in $55 \%$ of the 1028 estimations. The majority of misclassifications were by only one photo. Moreover, under- and overestimation was well balanced, except for rice and couscous. For rice there were underestimations of $39.4 \%$ vs. $19.7 \%$ for overestimations. For couscous $40.0 \%$ of the estimations were underestimations vs. $22.4 \%$ overestimations.

\section{Evaluation of portion size estimation on group level}

Table 2 shows the average weight differences between the served and estimated portion sizes for each of the dishes. These absolute errors show that there are underestimations for five of the eight dishes presented. Two of these (rice and couscous) were statistically significant $(P<0.05)$. The portion sizes of both sauces and stiff cereal porridge were generally overestimated by the subjects. However, the differences in mean estimates are all quite small. The average difference ranged from a $-8.4 \%$ underestimation for couscous to $6.3 \%$ overestimation for liquid sauce.

\section{Influence of sample characteristics on portion size estimations}

Table 3 shows the results of logistical regression modelling. The independent variables pregnancy status and village were removed from the full model, since they had no significant association with the outcome variable. Subjects attending school were 1.92 times more likely to choose the correct photograph than those who did not attend school. The served portion sizes were clearly associated with the subjects' ability to choose the correct photo. More precisely, if a medium or large portion size was served, subjects were 2.06 and 2.67 times more likely to choose an incorrect photo compared with the smallest served portion size. For the largest or the smallest portion size, misclassifications were equal.

Table 2 Comparison between the actual served portion sizes and the estimated portion sizes using food photographs

\begin{tabular}{|c|c|c|c|c|c|c|c|c|}
\hline \multirow[b]{2}{*}{ Food item } & \multirow[b]{2}{*}{$n$} & \multicolumn{2}{|c|}{ Actual weight $(g)$} & \multicolumn{2}{|c|}{ Estimated weight (g) } & \multicolumn{2}{|c|}{ Difference (g) } & \multirow[b]{2}{*}{$\%$ Differencet } \\
\hline & & Mean & SD & Mean & SD & Mean & SE & \\
\hline Stiff cereal porridge & 125 & 696.6 & 315.3 & 701.1 & 319.3 & 4.5 & 11.0 & 0.6 \\
\hline Rice & 132 & 434.8 & 177.1 & 407.0 & 172.0 & $-27.9^{\star}$ & 13.1 & -6.4 \\
\hline Rice and beans & 132 & 540.0 & 180.0 & 512.7 & 175.0 & -27.3 & 14.4 & -5.1 \\
\hline Couscous & 125 & 401.9 & 177.9 & 368.0 & 178.0 & $-33.9^{\star}$ & 13.0 & -8.4 \\
\hline Bean leaf balls & 125 & 453.4 & 147.3 & 437.3 & 145.0 & -16.1 & 9.8 & -3.6 \\
\hline Sauce, leafy & 125 & 238.6 & 134.3 & 248.2 & 148.8 & 9.6 & 7.1 & 4.0 \\
\hline Sauce, liquid & 132 & 196.4 & 100.4 & 208.6 & 98.0 & 12.3 & 7.5 & 6.3 \\
\hline Gruel & 132 & 412.7 & 176.1 & 401.8 & 178.6 & -10.9 & 11.2 & -2.6 \\
\hline
\end{tabular}

SD - standard deviation; SE - standard error.

${ }^{*}$ Difference is significant, $P<0.05$.

$\dagger \%$ Difference $=[($ mean actual weight - mean estimated weight $) /$ mean actual weight $] \times 100$.

Table 3 Results from the full model and reduced model in binary logistic regression analyses

\begin{tabular}{|c|c|c|c|c|}
\hline \multirow[b]{2}{*}{ Independent variable } & \multicolumn{2}{|c|}{ Full model } & \multicolumn{2}{|c|}{ Reduced model } \\
\hline & OR $(95 \% \mathrm{Cl})$ & $P$-value & OR $(95 \% \mathrm{Cl})$ & $P$-value \\
\hline \multicolumn{5}{|l|}{ Village } \\
\hline Kohot & 1.00 & & & \\
\hline Karaba & $0.82(0.64,1.05)$ & 0.12 & - & - \\
\hline \multicolumn{5}{|l|}{ Pregnancy status } \\
\hline Not pregnant $†$ & 1.00 & & & \\
\hline Pregnant & $1.05(0.81,1.36)$ & 0.70 & - & - \\
\hline \multicolumn{5}{|l|}{ School attendance } \\
\hline Yest & 1.00 & & 1.00 & \\
\hline No & $0.52(0.38,0.72)$ & $<0.001$ & $0.51(0.37,0.70)$ & $<0.001$ \\
\hline \multicolumn{5}{|l|}{ Portion size } \\
\hline Smallestt & 1.00 & & 1.00 & \\
\hline Medium & $2.06(1.40,3.03)$ & $<0.001$ & $2.06(1.40,3.03)$ & $<0.001$ \\
\hline Large & $2.67(1.86,3.84)$ & $<0.001$ & $2.67(1.85,3.83)$ & $<0.001$ \\
\hline Largest & $1.36(0.93,2.00)$ & 0.11 & $1.36(0.93,1.99)$ & 0.11 \\
\hline
\end{tabular}

OR - odds ratio; $\mathrm{Cl}$ - confidence interval.

†Reference class. 


\section{Discussion}

The validity of food photographs as a portion size estimation tool was evaluated for eight principal dishes of the central west of Burkina Faso. These foods have in common that they do not come in discrete units and hence it is more difficult to describe their quantity. Therefore food items such as fruits, bread and meat were not chosen. The quantity of such items is estimated more conveniently by counting the natural units 5 .

African women in rural areas generally eat from a shared bowl. This cultural habit can create additional difficulties in estimating the individual consumption pattern ${ }^{17}$ and in using food photographs with individual portion sizes. Therefore it should be recommended to ask participants of dietary assessment surveys in advance to eat off separate dishes during the recording period.

The objective and procedures of this study were explained in detail to the participants prior to their participation. This way of working could have a beneficial influence on the estimation ability when participants know that their collaboration is requested. Moreover, this aspect can perturb the performance of using food photographs in a dietary assessment survey if participants are not informed in advance of such a survey. In future dietary assessment studies planned in the context of our research, the protocol describes a first informative visit to the respondents before the 24-hour dietary recalls are undertaken.

The assessment of food portion sizes from photographs consists of three elements: perception, conceptualisation and memory. An extensive review of these elements has been given elsewhere ${ }^{8}$. Some previous studies have specifically evaluated the stage of perception ${ }^{6,8,13,18}$ or perception combined with conceptualisation ${ }^{4,9}$, while others have evaluated all three stages ${ }^{3-5,11}$. In the present study, perception, conceptualisation and memory over one day were included because the authors wanted to create a setting approximate to that of a 24-hour dietary recall.

Because of apparent differences in design, it is difficult to compare our findings with those from other studies. In urban South Africa Venter et al. ${ }^{13}$ reported 68\% correct answers when evaluating only the perception skills of the subjects, and other studies report matching percentages that do not exceed $70 \%{ }^{5-7,13}$. More important is that, in the present study, the percentages of under- and overestimation were quite balanced except for couscous and rice, where a slight but significant underestimation was noticed. Most previous studies do not report a systematic bias in photo-aided portion size estimation ${ }^{5,6,11-13}$ except for Nelson et al. ${ }^{9}$ and Frobisher and Maxwell ${ }^{4}$, who suggest a slight tendency to overestimation.

When our results are disaggregated per served portion size (results not shown) we see that the smallest portion is overestimated for all of the dishes, whereas the largest portion size is underestimated. This 'flat slope phenomenon' has been reported by other authors $3,8,10,13$. However, much depends on whether a subject is given the opportunity to choose a portion size smaller or larger than the largest depicted size. In our study, subjects were given the opportunity to choose a virtual portion size between or outside the range of the depicted portions, but only in $1.9 \%$ of the estimations was such portion size chosen. In South Africa, Venter et $a l^{13}$ mentioned that none of their subjects chose a portion outside the presented range although they were given the opportunity. This could be one explanation for the observed phenomenon.

The standard errors of the differences between served and estimated portion sizes reveal a large individual variation in the estimation capabilities of the subjects for the different dishes. This may give rise to some doubt over whether food photographs are a good tool as an instrument to estimate portion size on an individual level. Indeed, several studies report considerable random errors ${ }^{5,6,11,12}$ which are inconsistent in magnitude and/ or direction ${ }^{11}$. However, the average difference for the group of subjects shows rather small differences in absolute weight. Therefore we judge that food photographs are an appropriate tool to estimate portion size in studies that evaluate food consumption data at population level. Other authors like Robson and Livingstone ${ }^{11}$ validated food photographs in the framework of a 24-hour dietary recall and found that most nutrients were estimated to within $\pm 10 \%$ of intake. Pietinen et al. ${ }^{19}$ noticed that the use of food photographs increased the agreement between a quantitative FFQ and a weighed record.

From the results presented here we cannot deduce any specific reason why there was a discrepancy in the findings for rice and couscous. Both food items are amorphous and were served on a plate. There is no agreement in the literature on the influence of food shape on the portion size estimation abilities of participants using photographs. Robson and Livingstone ${ }^{12}$ found the best estimations for orange juice, whereas the portion size estimation for cheese was $32.8 \%$ higher. Venter et $a l^{13}$ reported that in their study solid foods were more accurately estimated than amorphous foods. In the study of Nelson et al. ${ }^{9}$ participants had more problems with estimating chips, mashed potatoes and spaghetti than cornflakes. Lillegaard et al. ${ }^{6}$ found the best results for mashed potatoes, pizza, meat, sauce, salad and cornflakes. During pre-testing the first version of our food album, subjects had severe problems with the portion size estimation of stiff cereal porridge pieces. Indeed, due to the great variety of traditional household measures used by the participants, pieces of stiff cereal porridge varied considerably in shape and geometry which made it very difficult to use one photographic series. Therefore it was decided to harmonise the shape of the served stiff cereal porridge pieces to facilitate a better estimation. However, 
this approach could induce a change in the consumed portion sizes when adopted in a dietary assessment survey. Lillegaard et al. ${ }^{6}$ reported similar problems for pizza when the served pizza differed from the form depicted in the photographs. Lucas et al. ${ }^{7}$ suggested that if the real food differs from the one on the photos in thickness of slices, number of food units or distribution on the plate, subjects had difficulties estimating the correct portion size.

Although previous studies have evaluated several population characteristics in relation to portion size estimates, no consistent picture emerges. Beasley et al. ${ }^{18}$ found significant differences in portion size estimates when schoolchildren assessed their usual portion size before or after lunch. However, not all subjects were affected by the level of satiety and it was suggested that the error could balance out in large field trials. Robinson et $a l^{10}$ found lower portion size estimation errors if subjects served themselves the portion instead of being served a portion. Nelson et al..$^{8}$ suggested that the accuracy of portion estimation could be influenced by age, gender, body mass index (BMI) and portion size. However, other authors did not find age and gender to be confounding factors ${ }^{3,11,13}$. In addition, in a large scale field trial with 448 male and female participants (6-60 years), Turconi et $a l^{12}$ reported no influence of age, gender or BMI on the portion size estimation ability of their subjects.

In our study, subjects who attended school were 1.92 times more likely to choose the correct photograph. One could postulate that educational level can influence a person's conceptualisation and memory performance and hence improve the ability to conceptualise and remember a photographic image. Another explanation could be that more educated subjects are more used to eating from individual plates. However, in this work no information was gathered on the relationship between school attendance and eating culture. Venter et $a l^{13}$ found that education had no influence on the portion size estimation ability of their subjects.

The variable pregnancy status was included because we intend to carry out future food consumption studies in the framework of an intervention study in this population. Pregnancy, however, proved to have no significant influence on the estimation abilities of our subjects.

We tried to imitate as much as possible the conditions of a 24-hour dietary recall study. During one day, two foods were served during the morning and another two foods were served in the evening, and the next day the portion size estimations were performed in the morning. We did not create an artificial testing site, but worked at the homes of the subjects. Therefore we believe that the results obtained can be largely extrapolated to a 24-hour dietary recall setting performed in the same environmental setting. A limitation of this study is that the design does not permit us to disentangle the different possible sources of errors reported by Nelson et $a l^{8}$ Therefore more research is warranted to evaluate influences of BMI, age, type of receptacle, and time elapsed between the consumption of the food and the estimation.

In conclusion, in spite of large individual errors, the results of this study suggest that food portion photographs could be used as an instrument to estimate portion sizes of frequently consumed foods on a group level in a rural setting. Further research could elucidate the influence of using photos for portion size estimation on quantitative food intake data.

\section{Acknowledgements}

The authors wish to acknowledge the financial support of the Flemish Interuniversity Council and Nutrition Third World. Our gratitude goes to the field team of the project and the many collaborators of this study. We thank the Centre Muraz for providing facilities for this collaborative research.

\section{References}

1 Cypel YS, Guenther PM, Petot GJ. Validity of portion-size measurement aids: a review. Journal of the American Dietetic Association 1997; 97: 289-92.

2 Bingham S, Nelson M, Paul P, Haraldsdottir J, Loken E, Van Staveren W. Methods for data collection at an individual level. In: Cameron M, Van Staveren W, eds. Manual on Methodology for Food Consumption Studies. Oxford: Oxford Medical Publications, 1988; 53-106.

3 Faggiano F, Vineis P, Cravanzola D, Pisani P, Xompero G, Riboli E, et al. Validation of a method for the estimation of food portion size. Epidemiology 1992; 3: 379-82.

4 Frobisher C, Maxwell SM. The estimation of food portion sizes: a comparison between using descriptions of portion sizes and a photographic food atlas by children and adults. Journal of Human Nutrition and Dietetics 2003; 16: 181-8.

5 Haraldsdottir J, Tjonneland A, Overvad K. Validity of individual portion size estimates in a food frequency questionnaire. International Journal of Epidemiology 1994; 23: 786-96.

6 Lillegaard IT, Overby NC, Andersen LF. Can children and adolescents use photographs of food to estimate portion sizes? European Journal of Clinical Nutrition 2005; 59: 611-17.

7 Lucas F, Niravong M, Villeminot S, Kaaks R, Clavelchapelon F. Estimation of food portion size using photographs validity, strengths, weaknesses and recommendations. Journal of Human Nutrition and Dietetics 1995; 8: 65-74.

8 Nelson M, Atkinson M, Darbyshire S. Food photography I the perception of food portion size from photographs. British Journal of Nutrition 1994; 72: 649-63.

9 Nelson M, Atkinson M, Darbyshire S. Food photography 2. Use of food photographs for estimating portion size and the nutrient content of meals. British Journal of Nutrition 1996; 76: 31-49.

10 Robinson F, Morritz W, McGuiness P, Hackett AF. A study of the use of a photographic food atlas to estimate served and self-served portion sizes. Journal of Human Nutrition and Dietetics 1997; 10: 117-24. 
11 Robson PJ, Livingstone MB. An evaluation of food photographs as a tool for quantifying food and nutrient intakes. Public Health Nutrition 2000; 3: 183-92.

12 Turconi G, Guarcello M, Berzolari FG, Carolei A, Bazzano R, Roggi C. An evaluation of a colour food photography atlas as a tool for quantifying food portion size in epidemiological dietary surveys. European Journal of Clinical Nutrition 2005; 59: 923-31.

13 Venter CS, MacIntyre UE, Vorster HH. The development and testing of a food portion photograph book for use in an African population. Journal of Human Nutrition and Dietetics 2000; 13: 205-18.

14 Burley V, Cade J, Margetts B, Thompson R, Warm D. Consensus Document on the Development, Validation and Utilisation of Food Frequency Questionnaires. Report No. ANO850. Leeds: Ministry of Agriculture, Fisheries and Food, 2000; 1-62.
15 Lykke AM, Mertz O, Ganaba S. Food consumption in rural Burkina Faso. Ecology of Food and Nutrition 2002; 41: 119-53.

16 Sim J, Wright CC. The kappa statistic in reliability studies: use, interpretation, and sample size requirements. Physical Therapy 2005; 85: 257-68.

17 Hudson GJ. Food intake in a West African village. Estimation of food intake from a shared bowl. British Journal of Nutrition 1995; 73: 551-69.

18 Beasley LJ, Hackett AF, Maxwell SM, Stevenson L. The effect of a dietary preload on estimation of usual food portion size by photograph in female volunteers. Journal of Human Nutrition and Dietetics 2004; 17: 219-25.

19 Pietinen P, Hartman AM, Haapa E, Rasanen L, Haapakoski J, Palmgren J, et al. Reproducibility and validity of dietary assessment instruments 1. A self-administered food use questionnaire with a portion size picture booklet. American Journal of Epidemiology 1988; 128: 655-66.

Appendix A - The weight of the portion size depicted in each food photograph

\begin{tabular}{lcccc}
\hline & \multicolumn{3}{c}{ Depicted weight $(\mathrm{g})$} \\
\cline { 2 - 5 } Food item & Photo 1 & Photo 2 & Photo 3 \\
\hline Stiff cereal porridge & 280 & 560 & 840 & Photo 4 \\
Rice & 200 & 360 & 520 & 1120 \\
Rice and beans & 300 & 460 & 620 & 680 \\
Couscous & 160 & 320 & 480 & 780 \\
Bean leaf balls & 260 & 390 & 300 & 640 \\
Sauce, leafy & 60 & 180 & 240 & 650 \\
Sauce, liquid & 60 & 150 & 500 & 330 \\
Gruel & 180 & 340 & & 660 \\
\hline
\end{tabular}

\section{Appendix B - Examples of the food photograph series used}

\section{Series 1: Liquid sauce}

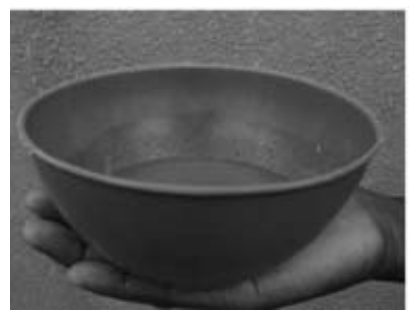

Photo 1

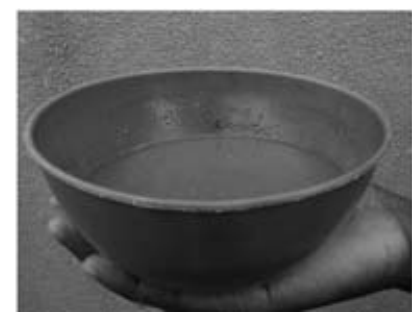

Photo 2

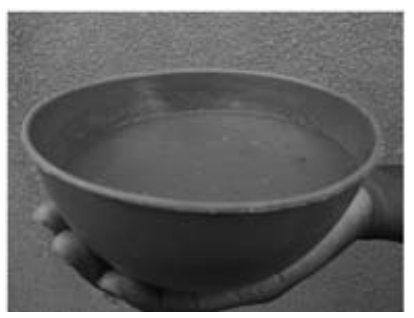

Photo 3



Photo 4

\section{Series 2: Rice and beans}

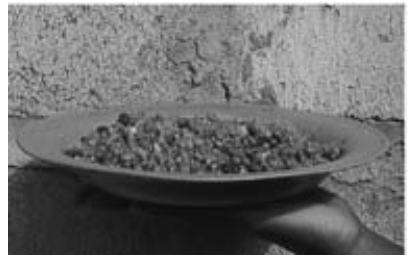

Photo 1



Photo 2



Photo 3

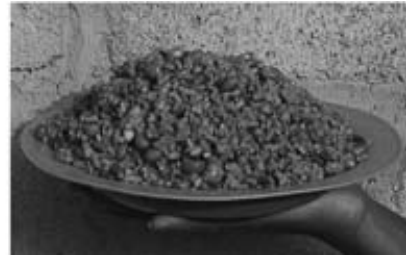

Photo 4 